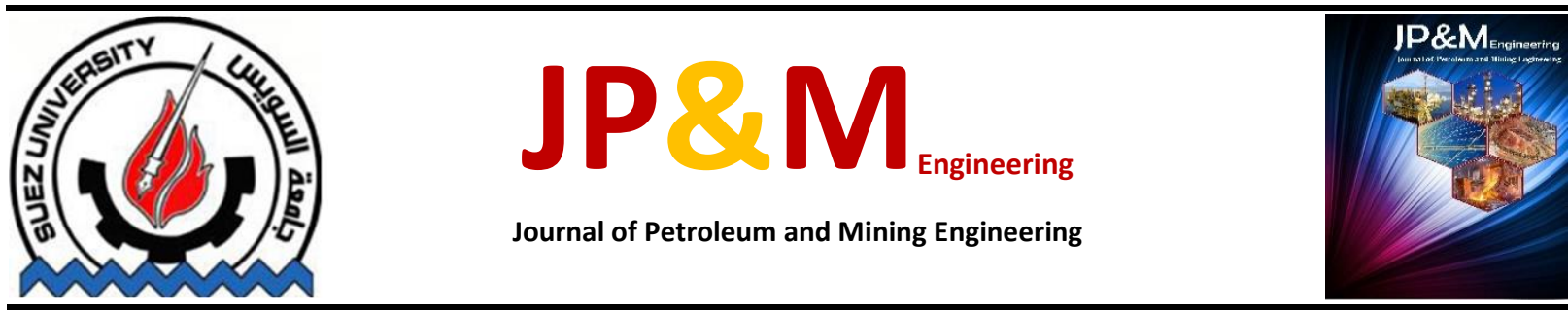

\title{
Surface Mechanical Attrition Treatment of Commercially Pure Titanium Using Ball Milling Attritor
}

\author{
Ahmed E. Hannora ${ }^{\text {a* }}$ and M.M.Z. Ahmed ${ }^{\mathrm{a}, \mathrm{b}}$ \\ ${ }^{a}$ Faculty of Petroleum and Mining Engineering, Suez University, Suez, 43512, Egypt. \\ ${ }^{\mathrm{b}}$ The British University in Egypt \\ *Corresponding authore-mail: ahmed.hannora@suezuniv.edu.eg \\ $+20147043930$
}

\section{Keywords}

Titanium-VT1-0;surface mechanical attrition treatment;hardness; attritor

\section{Introduction}

Titanium has unique properties which make it attractive to designers in a variety of applications such as aerospace, chemical processing, power generation and automotive. Also the biomedical applications of titanium receives a great interest which have benefits of the metal's inertness for use in surgical implants and prosthetic devices in human body [1-4]. The main disadvantage of the commercially pure titanium as a structural material is its low characteristics of strength both under static and dynamic loads as compared with the other alloys based on titanium. Also it is widely recognized that titanium displays poor wear resistance and that its fatigue performance depends to a large extent on its surface properties [3].

Optimization of the surface properties of materials is of great concern at the present time since most failures start from the surface (such as fatigue, fretting corrosion, corrosion, and wear). As a result, improving the surface properties would greatly enhance the overall behaviour of materials. The introduced mechanical treatment process has a considerable technological importance; it improves the surface properties of titanium. Consequently, extended applications of high levels requirements of reliable performance can be anticipated in aerospace, automotive, chemical plant, power generation in addition to surgery and medicine and

\begin{abstract}
The influence of surface mechanical attrition treatment (SMAT), as a nonconventional solid-state process, on the surface of Ti-VT1-0 substrate has been optical microscopy, X-Ray Diffraction (XRD), and Vicker's hardness measurement. A highly deformed layer, like coated layer, was observed at the surfaces of the substrates. This layer consists of fine grain structure with no clear variation with the AT time. However the hardness measurement showed a systemic behavior of increasing by increasing SMAT time up to $60 \mathrm{~min}$. A drop in hardness values is associated with further treatment time up to $240 \mathrm{~min}$. XRD results were consistent with the hardness behaviour and showed a notable broadening and peak intensity reduction with increasing SMAT time up to $60 \mathrm{~min}$ followed by a peak intensity increases by increasing SMAT time up to $240 \mathrm{~min}$.
\end{abstract}

other major industries. Mechanical surface treatments results in a high level of dislocation densities in near-surface regions; this may happen as a result of the local plastic deformation and residual stresses that developed [5-7]. Thus the aim of the current work was to examine the effect of SMAT using ball milling attritor on the surface properties of commercial pure titanium.

\section{Materials and Methods}

The starting material was commercially pure titanium VT1-0 substrates of dimensions $10 \times 10 \times 3$ $\mathrm{mm}$. SMAT was carried out using relatively high energy ball milling attritor of $500 \mathrm{rpm}$ rotating speed in static air. Hardened steel balls of $3 \& 5 \mathrm{~mm}$ diameter were placed in the O-ring sealed vial of the attirator with Ti substrate. The ball to substrate ratio was 10:1. SMAT were carried out for various times of $30,60,120$ and 240 minutes. Nearly $1 / 5$ of the vial volume was filled. For optical microscopy investigation the thin cross section of the substrates was prepared according to the standard metallographic preparation by grinding, mechanical polishing and etching with bakers reagent that consists of $96 \mathrm{ml}$ distilled water, $2 \mathrm{ml} \mathrm{HF}$ and $2 \mathrm{ml}$ $\mathrm{HNO}_{3}$.

XRD was performed using D5000 powder diffractometer using $\mathrm{Cu} K \alpha$ radiation (wavelength $\lambda=$ $0.15406 \mathrm{~nm}$ ) with a nickel filter at $40 \mathrm{kV}$ and $30 \mathrm{~mA}$. The diffractometer was operated within range of 
$30<2 \theta<80$ with step-time $=3$ seconds and step-size $=$ 0.05 degree. Diffraction signal intensity throughout the scan was monitored and processed with DIFFRACplus software. Topas 2.1 Profile fit software was used to extract the peak parameters and the Pseudo Voigt function was used to model the peak shape. Vicker's hardness was determined on the large surface of the Ti substrates - using $200 \mathrm{gm}$ load in HWDV-75 Vickers hardness testing machine. Five measurements were taken on each surface of the substrate and the average was considered.

\section{Results and Discussion}

This section may be divided by subheadings. You can apply "Heading 2 and Heading 3 " if needed. It should provide a concise and precise description of the experimental results, their interpretation as well as the experimental conclusions that can be drawn. Authors should discuss the results and how they can be interpreted in perspective of previous studies and of the working hypotheses. The findings and their implications should be discussed in the broadest context possible. Future research directions may also be highlighted.

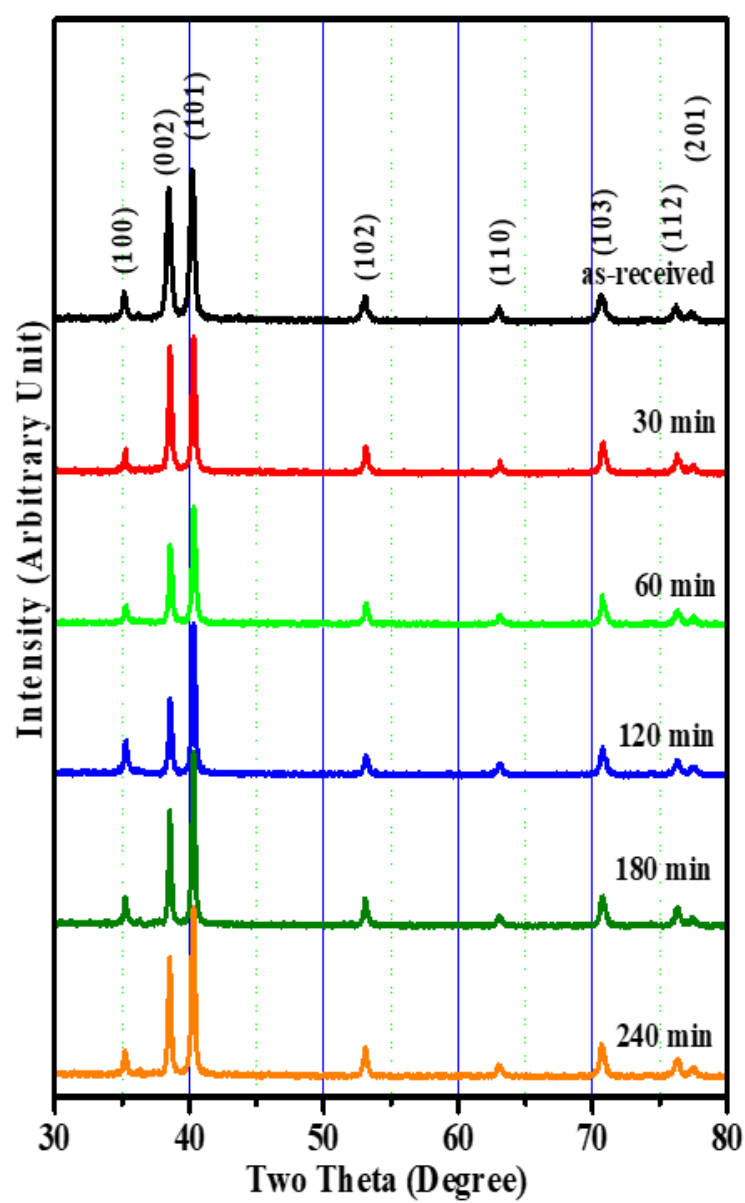

Figure 1 XRD patterns of surface mechanical treatment Ti-substrate with time

Figure 1 shows the XRD of the titanium samples subjected to SMAT for various times. With increasing surface mechanical treatment time up to 60 min., the XRD patterns show a notable intensity reduction of (002) and (101) peaks with the (002) peak shows intensity reduction much more than the other peak.
Then by increasing SMAT time to $120 \mathrm{~min}$, the (101) peak starts to increase again while the (002) peak with the same intensity as that of $60 \mathrm{~min}$. Increasing the SMAT up to 180 and 240 min the intensity of both (002) and (101) peaks increase again. From this observation it can be noted that the transition of (002) from reduction to increase again occurs after 180min SMAT time, while that of (101) peak occurs after 120min SMAT time. This would suggest a difference in the deformation mechanism operating in each of the two planes. According to Crocker and Bevis [8] cold working deformation results of titanium show that twinning deformation occurs first and slip deformation operates at high strain levels. During SMAT, increasing time means increasing of accumulated strain on the substrate surface. So that it is expected to have twining deformation mechanism operating up to 60min SMAT and then slip deformation starts to operate starting from 120min time.

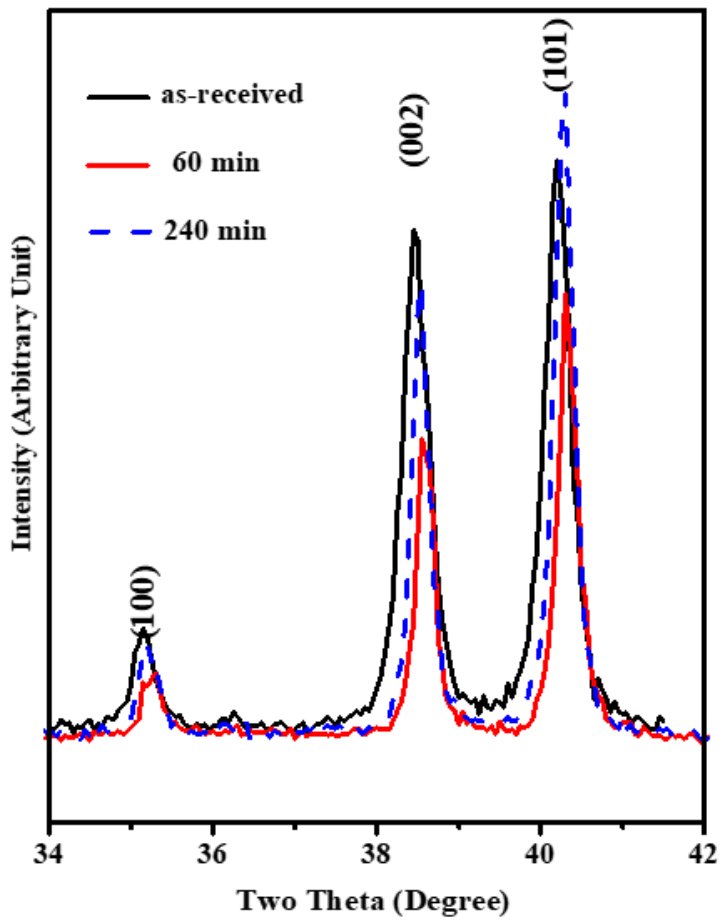

Figure 2 Uniformly shift at XRD patterns of surface mechanical treatment Ti-substrate with time.

Figure 2 shows zoomed-in view of the XRD patterns after 60 and $240 \mathrm{~min}$, the line shift (peak position) could be due to effect of uniform strain during the SMAT process. Also, the area under the peaks decreases after $60 \mathrm{~min}$ of SMAT showing crystallinity reduction then increases with SMAT time as a result of increasing thermal energy.

The uniform strain in the deformed surface layer can be accessed from $x$-ray diffraction patterns as a function of SMAT time. Figure 3 shows the variation of the peaks position with the SMAT time. This is clearly reflects the amount of accumulated strain effect on the various crystallographic planes. It is observed that twinning could contribute significantly to accommodation of the deformation at low strains, Twinning could accommodate only a portion of the plastic strains in the fan-shaped deformation zone. Even in this zone, by dislocation slip, a significant 
portion of the strains are expected to be accommodated which results in some refinement of the microstructure. Ti has an hcp crystal structure with a $\{10 \overline{1} 0\}\langle 11 \overline{2} 0\rangle$ slip system at room temperature. Its slip systems changes to $\{0001\}\langle 11 \overline{2} 0\rangle$ at the temperature range $\left(400-450^{\circ} \mathrm{C}\right)$ [1]. Generally, the number of slip systems - which is equivalent to the number of dislocation glide opportunities in a crystal lattice - is only 3 for the hcp structure. The denser slip planes are packed with atoms, the easier dislocations can glide [1]. Compared with ICCD standard titanium patterns (PDF number 44-1294), no secondary phases were present. Figure 4 shows the variation of unit cell dimensions, where the unit cell volume decreases after 60 minute of SMAT and then increases again. This increase in unit cell dimension could be due to sever plastic deformation and strain accumulation.

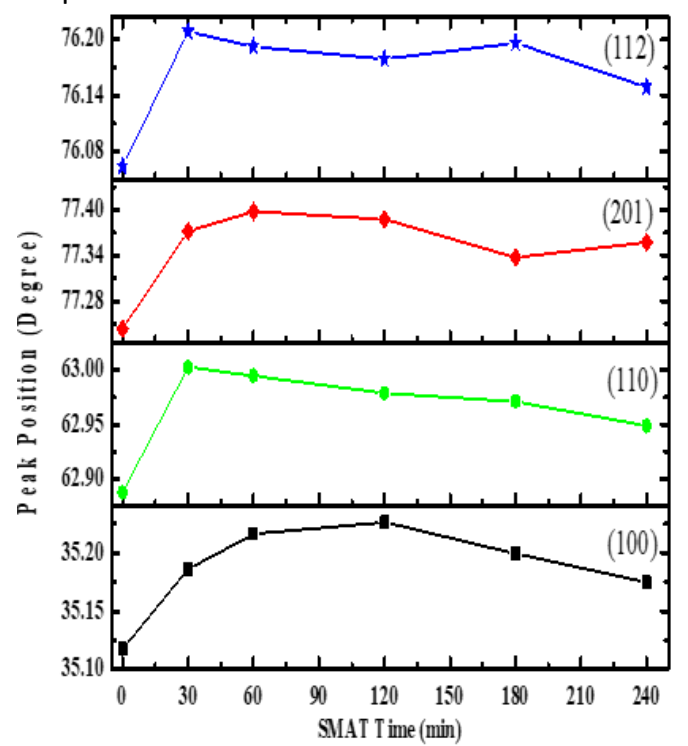

Figure 3 Variation of the peak position of surface mechanical treatment Ti- peaks with time.

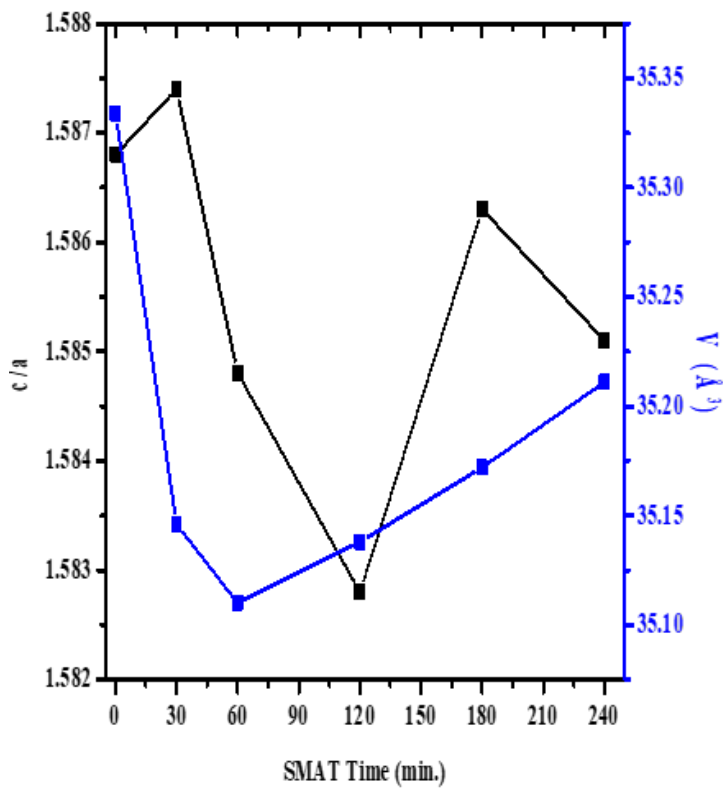

Figure 4 Variation of the unit cell dimension of surface mechanical treatment $\mathrm{Ti}$ - peaks variation with time.
SMAT. It can be observed that SMAT results in a significant refining of grain structure at the surface in a depth of about $100 \mu \mathrm{m}$. In addition to the grain refining, another microstructural feature can be observed that is the twins that formed inside the grains. During SMAT the surface of the titanium substrate subjected to sever plastic deformation with high strain and strain rate. The sever plastic deformation results in a great amount of accumulated strain which surely will affect the surface microstrctural features. The same features were found with SEM, Figure 6.

The microstructural changes that occur in titanium surface during SMAT was reported by Zhu et at [3] in the following sequence. (1) the onset of twins and the intersection of twin systems, (2) the formation of low angle disoriented lamellae displaying a high density of dislocations, (3) the subdivision of microbands into blocks and the resulting formation of polygonal submicronic grains and (4) The further breakdown of submicronic polygonal grains into nano-grains. The first three stages in this sequence of microstructural changes are expected to be occurred during SMAT using the current technique and the optical micrographs confirm that. However the existence of nano-grains requires TEM investigation in order to be detected. In comparison to the other techniques of SMAT used in the literature [9], the current technique has a unique advantage that the surface treatment takes place in all sides of the substrate. Also it is reported that, [10] repeated peening loads generate more dislocations which will annihilate and recombine into small angle grain boundaries separating subgrains. These subgrains still contain high density of dislocations, and can be further refined to nano grains through the development of the dislocations in the subgrains.

In order to investigate the effect of SMAT on the mechanical properties of the Ti-substrates, Vickers hardness values were measured on the surface of the substrate before and after SMAT. Figure 7 illustrates the variation of vicker's hardness values with the time of SMAT. Clearly it can be observed that hardness increases after SMAT, however there is a peak for the hardness after 60min of SMAT after which the hardness starts to decrease again but still with values higher than the as received material hardness. It should be noted here that, the hardness results is consistent with the XRD chart results explained above, i.e., the shift of the two peaks intensity from reduction to increase accompanied with the shift of the hardness from increase to reduction. This behavior also can be interpreted with the change in the deformation mode from twinning at low strains to slip at high strain values. Also the contribution of temperature rise due to more collisions between samples and balls

Figure 5 shows the optical microstructure of the titanium substrate cross section before and after 


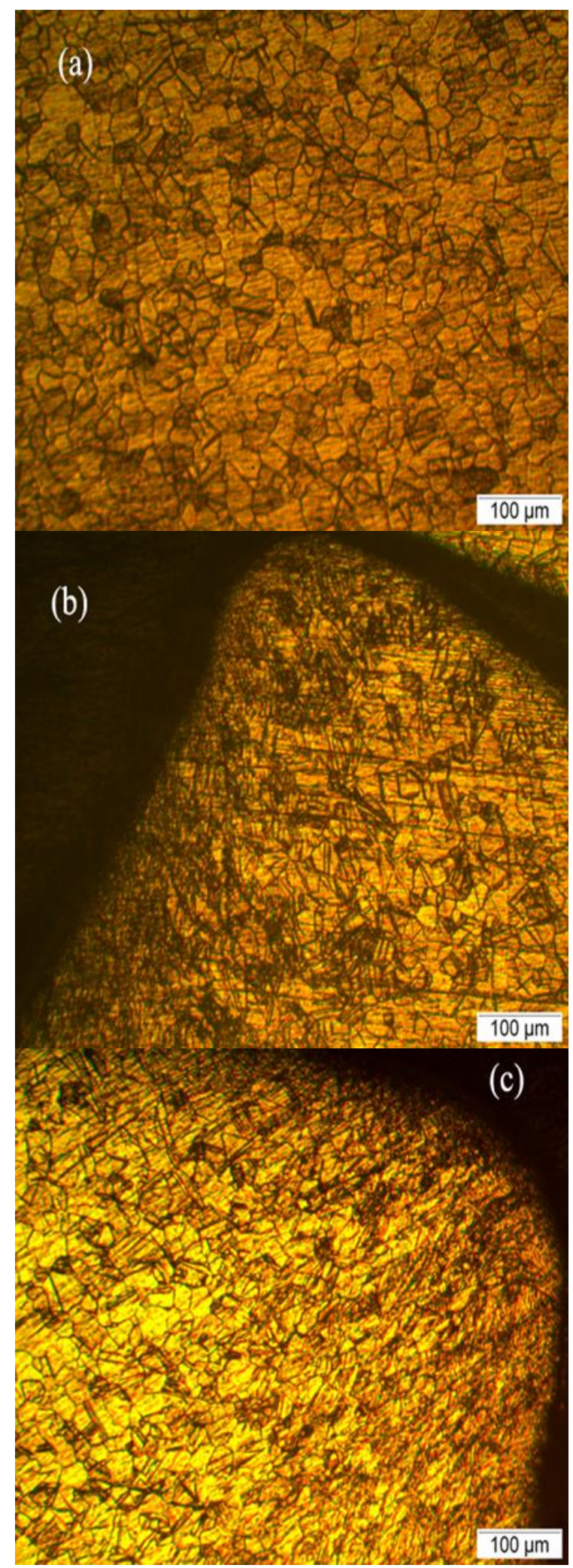

Figure 5 Optical microstructure of commercial pure titanium a) as recived, b) after SMAT for 30min and b) after SMAT for 60mi

\section{Conclusions}

From the current investigation of the effect of SMAT on the properties of commercially pure $\mathrm{Ti}$, the following conclusions can be drawn:-

- SMAT of pure Ti using the ball mill attritor is an effective technique for the surface treatment with a unique advantage of all sides can be treated in the same time.
- Increasing of SMAT time after 60 min results in hardness reduction after hardness peak at that time. This change of hardness was consistent with the change of XRD peak intensities from reduction to increase again.

- Significant grain refining has occurred on the titanium surface after SMAT using the current SMAT technique.

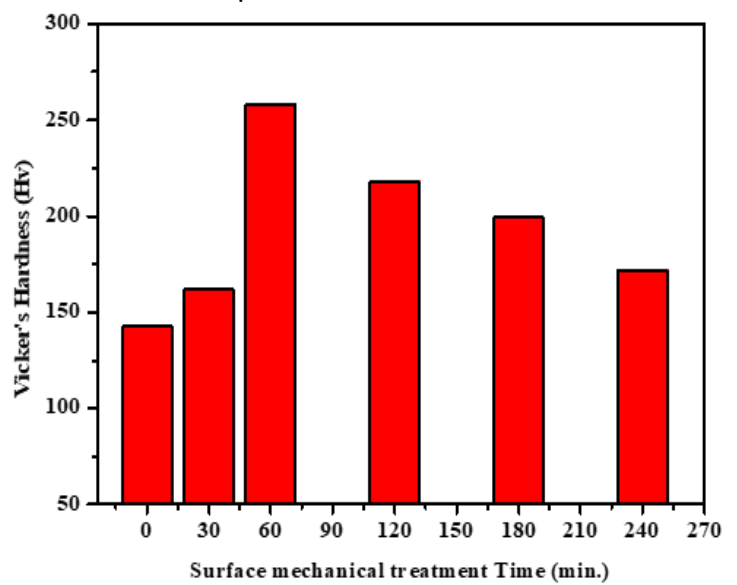

Figure 6 Variation of titanium Vicker's hardness with surface mechanical treatment time.

\section{References}

[1] Lutjering G. and Williams J.C. "Engineering materials and processes: Titanium" 2nd edition, Springer, New York (2007).

[2] Wu S., Fan K., Jiang P., Chen. S., "Grain refinement of pure Ti during plastic deformation." Materials Science and Engineering A 527 (2010), 6917-6921.

[3] Markovs'kyi P. E., Pishchak V. K., Mordyuk B. M. and Okrainets P. N. "Improvement of the fatigue characteristics of VT1-0 titanium alloy by the surface mechanical and rapid thermal treatment." Materials Science 42, 3 (2006), 376-383.

[4] Zhu K.Y., Vassel A., Brisset F., Lu K., Lu J., Acta Materialia 52, 14 (2004), 4101-4110.

[5] Torosyan A., Tuck J., Korsunsky A., Bagdasaryan S. A, Materials Science Forum, 386-388, (2002), 251-256.

[6] Wagner, L. "Mechanical surface treatments on titanium, aluminum and magnesium alloys." Materials Science and Engineering A 263 (1999), 210-216.

[7] Ravi Shankar M., Rao B.C., Lee S., Chandrasekar S., King A. H., Dale Compton W. "Severe plastic deformation (SPD) of titanium at near-ambient temperature." Acta Materialia 54, 14 (2006), 36913700 .

[8] Crocker AG, Bevis M. The science technology and application of titanium. Oxford: Pergamon Press; (1970).

[9] Lu K., Lu J. Surface nanocrystallization (SNC) of metallic materials-presentation of the concept behind a new approach. Journal of Materials Science and Technology, 15, 3 (1999), 193-197.

[10] Liu G., Lu J., Lu K. Surface nanocrystallization of 316L stainless steel induced by ultrasonic shot peening. Materials Science and Engineering A, 286 (2000), 9195. 